\title{
Cul1 promotes melanoma cell proliferation by promoting DEPTOR degradation and enhancing cap-dependent translation
}

\author{
LAN CHEN, TIANYU LIU, YUNHUA TU, DONGYUN RONG and YU CAO
}

Department of Dermatology, The Affiliated Hospital of Guiyang Medical University, Guiyang, Guizhou 550004, P.R. China

Received August 15, 2015; Accepted September 15, 2015

DOI: $10.3892 /$ or. 2015.4442

\begin{abstract}
Cullin1 (Cul1) serves as a rigid scaffold in the SCF (Skp1/Cullin/Rbx1/F-box protein) E3 ubiquitin ligase complex and has been found to be overexpressed in melanoma and to enhance melanoma cell proliferation by promoting G1-S phase transition. However, the underlying mechanisms involved in the regulation of melanoma cell proliferation by Cul1 remain poorly understood. In the present study, we found that Cul1 promoted mTORC1 activity and cap-dependent translation by enhancing the ubiquitination and degradation of DEPTOR. We further showed that suppression of the eIF4F complex assembly profoundly inhibited the promoting effect of Cul1 on melanoma cell proliferation, while enhancement of the eIF4F complex activity reversed the inhibitory effect of Cul1 depletion on melanoma cell proliferation, indicating that Cull contributes to melanoma cell proliferation by activating cap-dependent translation. These data elucidate the role of Cul1 in cap-dependent translation and improves our understanding of the underlying mechanisms involved in the regulation of melanoma cell proliferation by Cull.
\end{abstract}

\section{Introduction}

Melanoma, derived from epidermal melanocytes, represents the most serious type of skin cancer and accounts for $80 \%$ of skin cancer-related deaths (1). Cul1, an essential scaffold of the SCF (Skp1/Cullin/Rbx1/F-box protein) E3 ubiquitin ligase complex, has been reported to be overexpressed in many cancer tissues and is significantly correlated with the

Correspondence to: Professor Yu Cao, Department of Dermatology, Affiliated Hospital of Guiyang Medical Univesity, 28 Guiyi Street, Yunyan, Guiyang, Guizhou 550004, P.R. China

E-mail: caoyudoctor1@aliyun.com

Abbreviations: Cul1, Cullin1; SCF, Skp1/Cullin/Rbx1/F-box protein; eIF4E, eukaryotic translation initiation factor 4E; 4E-BP1, eIF4E-binding protein 1; p70S6K, ribosomal p70 S6 kinase; mTORC1, mammalian target of rapamycin complex 1 .

Key words: cap-dependent translation, Cul1, melanoma, DEPTOR, mTORC1, 4E-BP1, cell proliferation poor prognosis of tumors, including hepatocellular carcinoma, colorectal cancer, glioma, lung cancer, breast cancer and gastric cancer (2-7). In melanoma, Cull expression is increased in the early stages of melanoma (8). Cul1, combined with BRG1, Bim and ING4, aid in the discrimination of melanoma from dysplastic nevi (9). Cull enhances melanoma cell proliferation by promoting G1-S phase transition (10). However, the underlying mechanisms involved in the regulation of melanoma cell proliferation by Cull remain poorly understood.

The eIF4F complex plays a critical role in cancer development by facilitating the cap-dependent translation of oncogenic mRNAs, such as cyclin D1, c-Myc, VEGF and Mcl (11). The eIF4F complex consists of eIF4A, eIF4G1 and eIF4E, and its assembly is largely dependent on eIF4E availability, which is negatively regulated by $4 \mathrm{E}-\mathrm{BP} 1$ phosphorylation (12). The unphosphorylated or hypophosphorylated 4E-BP1 binds to the eIF4E surface antagonistically with eIF4G and supprepresses the formation of the eIF4F complex. Phosphorylation of $4 \mathrm{E}-\mathrm{BP} 1$ causes $4 \mathrm{E}-\mathrm{BP} 1$ to disassociate from eIF4E and thus allows eIF4F assembly and translation initiation. In melanoma, hyperphosphorylated 4E-BP1 was reported to be associated with worse overall and post-recurrence survival (13).

The mammalian target of rapamycin complex 1 (mTORC1) phosphorylates 4E-BP1 on Thr37 and Thr46, which promotes subsequent phosphorylation of Ser65 and Thr70 and thus enhances cap-dependent translation (14). mTORC1 consists of mTOR, Raptor, PRAS40, G $\beta$ L and DEPTOR, one of its own endogenous inhibitors (15). DEPTOR inhibits mTORC1 activity through binding to the FAT domain of mTOR through its PDZ domain (16). Due to its inhibitory effect on mTORC1 activity, DEPTOR acts, in general, as a tumor suppressor by suppressing cap-dependent translation and cell proliferation. DEPTOR activity is regulated largely by the control of DEPTOR levels, which are negatively regulated by $\mathrm{SCF}^{\beta \operatorname{TrCP}}$ E3 ubiquitin ligase (17-19). By binding to DEPTOR, $\mathrm{SCF}^{\beta \mathrm{TrCP}}$ promotes the ubiquitination and degradation of DEPTOR, leading to activation of mTORC1. Given that Cull serves as a rigid scaffold in the SCF complex and aberrant expression of Cul1 results in dysfunction of SCF E3 ligases, we speculated that Cul1 may promote cap-dependent translation and melanoma cell proliferation by promoting DEPTOR degradation and enhancing mTORC1 activity.

In the present study, we investigated the effect of Cull on DEPTOR expression, mTORC1 activity and cap-dependent translation in melanoma cells. We found that Cull regulated 
mTORC1 activity through degradation of DEPTOR, which promoted 4E-BP1 phosphorylation and cap-dependent translation. Furthermore, we found that suppression of mTORC1 activity or the eIF4F complex assembly profoundly inhibited the promotive effect of Cull on melanoma cell proliferation, while enhancing the eIF4F complex activity by silencing the expression of 4E-BP1 significantly antagonized the inhibitory effect of Cull depletion on melanoma cell proliferation. Our data indicate that Cull promotes melanoma cell proliferation by promoting DEPTOR degradation and enhancing cap-dependent translation.

\section{Materials and methods}

Antibodies and reagents. Antibodies against P70S6K, pP70S6K (T389), 4EBP-1, p4EBP-1 (T37/46), p4EBP-1 (S65), cyclin D1, eIF4E and eIF4G were obtained from Cell Signaling Technology (Beverly, MA, USA). Antibodies from Santa Cruz Biotechnology (Santa Cruz, CA, USA) included Cull and tubulin. Antibody against DEPTOR was obtained from Millipore (Billerica, MA, USA). Anti-ubiquitin antibody was purchased from Sigma (St. Louis, MO, USA). 4EGI-1 and PP242 was provided by Calbiochem (Darmstadt, Germany) and Selleckchem (Houston, TX, USA), respectively. MG132 was obtained from Sigma.

Cells and cell culture. A375 and Mewo cells were cultured in Dulbecco's modified Eagle's medium (DMEM; Invitrogen, Carlsbad, CA, USA) containing 10\% fetal bovine serum (FBS; Hyclone, Logan, UT, USA) and 1\% penicillin/streptomycin. All treatments with 4EGI-1 were conducted in DMEM containing $5 \%$ FBS. Cells were maintained in a $37^{\circ} \mathrm{C}$ incubator at $5 \% \mathrm{CO}_{2}$.

For stable overexpression of Cul1, A375 and Mewo cells were transfected with the $\mathrm{pCMV}-2 \mathrm{~B}-\mathrm{Cul1}$ vector and control cells were transfected with the pCMV-2B backbone. Cells were transfected with Lipofectamine ${ }^{\mathrm{TM}} 2000$ (Invitrogen, Carlsbad, CA, USA) according to the manufacturer's instructions, and stable transformants were selected using $500 \mu \mathrm{g} / \mathrm{ml}$ G418 (Calbiochem).

To silence the expression of Cul1, A375 and Mewo cells were infected with appropriate amounts of lentiviral particles carrying control shRNA or Cull shRNA (GeneChem Co., Shanghai, China). Virus-containing medium was discarded and replaced with fresh medium after $12 \mathrm{~h}$. At $48 \mathrm{~h}$ post-infection, stable Cull-knockdown cells were selected in puromycin $(1 \mu \mathrm{g} / \mathrm{ml})$.

Immunoblotting. Cells were lysed in RIPA buffer $(150 \mathrm{mM}$ $\mathrm{NaCl}, 50 \mathrm{mM}$ Tris-HCl, $\mathrm{pH} 7.5,1 \%$ sodium deoxycholate, $0.1 \%$ SDS, 1 mM EDTA, 1 mM EGTA, and 1\% NP-40) containing protease inhibitors. Protein $(40-80 \mu \mathrm{g})$ was electrophoresed on $10 \%$ SDS-PAGE gel after measuring the protein concentration using the bicinchoninic acid (BCA) assay reagent (Pierce Chemical, Rockford, IL, USA) and then transferred to nitrocellulose membranes (Amersham Biosciences, Piscataway, NJ, USA). The membranes were blocked with $5 \%$ non-fat milk in $0.1 \%$ PBS-Tween for $2 \mathrm{~h}$ at room temperature and then incubated with primary antibodies overnight at $4^{\circ} \mathrm{C}$, followed by incubation with anti-rabbit/mouse/goat IgG conjugated to HRP for $2 \mathrm{~h}$ at room temperature. Detection was performed using the $\mathrm{ECL}^{\mathrm{TM}}$ Advance Western Blotting detection kit (GE Healthcare, Buckinghamshire, UK).

Ubiquitination assay. Cells were collected in lysis buffer (20 mM HEPES, pH 7.2, $50 \mathrm{mM} \mathrm{NaCl}, 0.5 \%$ Triton X-100, $1 \mathrm{mM} \mathrm{NaF}$ and $1 \mathrm{mM}$ DTT) supplemented with protease inhibitors. To detect endogenous DEPTOR ubiquitination, precleared cell lysates were incubated with the DEPTOR antibody with gentle rotation at $4^{\circ} \mathrm{C}$ for $2 \mathrm{~h}$, and then proteinA beads were added for an additional 2-h incubation at $4^{\circ} \mathrm{C}$ with gentle rotation. After being washed three times with lysis buffer, the precipitated beads were analyzed by immunoblotting using the ubiquitin antibody.

siRNA and transient transfections. siRNA for 4EBP1 and DEPTOR were purchased from Invitrogen. A375 and Mewo cells were transfected with 4EBP1 or DEPTOR siRNA or the negative control using Lipofectamine RNAiMAX (Invitrogen) according to the manufacturer's instructions. At $48 \mathrm{~h}$ post-transfection, the cells were lysed and subjected to assays for immunoblotting, cap-dependent translation and apoptosis. For the CCK- 8 assay, cells were seeded into 96-well plates at $18 \mathrm{~h}$ post-transfection.

$m^{7}$ GTP pull down assay. Cells were prepared in $\mathrm{m}^{7} \mathrm{GTP}$ lysis buffer containing $20 \mathrm{mM}$ Tris, $100 \mathrm{mM} \mathrm{KCl}, 20 \mathrm{mM}$ $\beta$-glycerophosphate, $1 \mathrm{mM}$ EGTA, $1 \mathrm{mM}$ EDTA, $1 \mathrm{mM}$ dithiothreitol, $1 \mathrm{mM}$ phenylmethylsulfonyl fluoride, $0.25 \mathrm{mM}$ $\mathrm{Na}_{3} \mathrm{VO}_{4}, 10 \mathrm{mM} \mathrm{NaF}$, and $1 \mathrm{X}$ protease inhibitor cocktail. For the $\mathrm{m}^{7} \mathrm{GTP}$ pull down assay, cell lysates (500 $\mu \mathrm{g}$ protein) were incubated with $30 \mu \mathrm{l}$ of $\mathrm{m}^{7} \mathrm{GTP}$-sepharose beads (GE Healthcare, Chalfont St. Giles, UK) for $3 \mathrm{~h}$ at $4^{\circ} \mathrm{C}$. Precipitates were washed three times with $500 \mu \mathrm{l}$ of phosphate-buffered saline containing $0.5 \mathrm{mg} / \mathrm{ml}$ of heparin and $2 \mathrm{mM} \mathrm{MgCl}_{2}$, and then analyzed by immunoblotting with the indicated antibodies.

Bicistronic luciferase assays. A375 or Mewo cells were transiently transfected with a bicistronic luciferase reporter plasmid, pcDNA3-rLuc-PolioIRES-fLuc, using Lipofectamine ${ }^{\mathrm{TM}} 2000$ following the manufacturer's instructions. This plasmid directs cap-dependent translation of the Renilla luciferase (RL) gene and cap-independent Polio IRES-mediated translation of the firefly (FL) gene. At $48 \mathrm{~h}$ post-transfection, the luciferase activity was measured with the Dual-Luciferase reporter assay kit (Promega, Madison, WI, USA) according to the manufacturer's instructions. Cap-dependent translational activity was determined by calculating the ratio of Renilla/firefly luciferase luminescence. Assays were performed in triplicate, and results are presented as means \pm standard deviation (SD).

Cell proliferation assays. Cells were seeded in 96-well plates $(2,000$ cells/well). At $18 \mathrm{~h}$ post-transfection, the cells were treated with the agents as indicated for $48 \mathrm{~h}$. After treatment, cell proliferation was detected using the Cell Counting Kit-8 (CCK-8; Dojindo Laboratories, Tokyo, Japan) assay according to the manufacturer's instructions, and optical density (OD) was measured at $450 \mathrm{~nm}$. The OD value of the treatment group was normalized to the values from the untreated control group. Assays were performed in triplicate, and the results are presented as means \pm standard deviation (SD). 
A

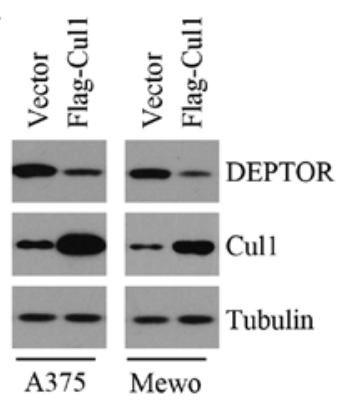

B

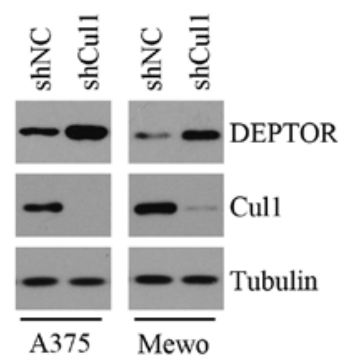

C

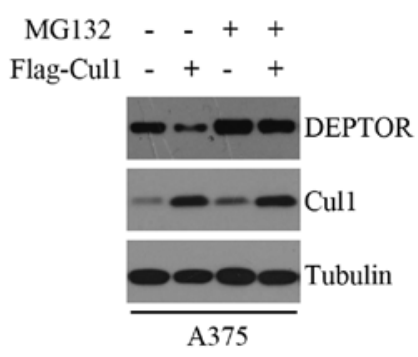

D

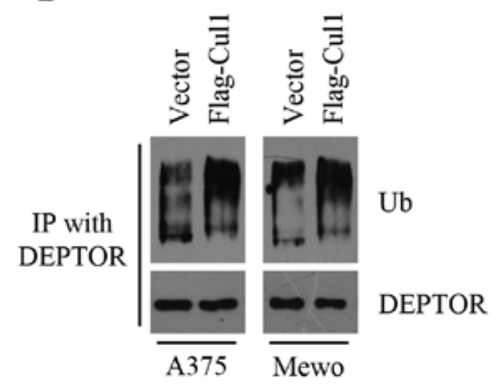

$\mathrm{E}$

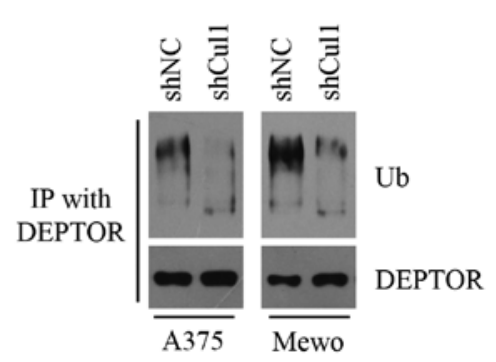

Figure 1. Cul1 promotes the ubiquitination and degradation of DEPTOR. (A) Cell lysates of control and melanoma cells (A375 and Mewo) stably transfected with FLAG-tagged Cull were prepared and immunoblotted with the indicated antibodies. Tubulin was used as a loading control. (B) Cell lysates of control and Cul1-depleted melanoma cells (A375 and Mewo) were prepared and immunoblotted with the indicated antibodies. Tubulin was used as a loading control. (C) Western blot analysis of control and A375 cells stably transfected with FLAG-tagged Cul1 treated with DMSO or $10 \mu$ M MG132. (D and E) Ubiquitination assays for DEPTOR. Cell lysates from A375 and Mewo cells stably expressing FLAG-tagged Cul1 (D) or Cul1-depleted A375 and Mewo cells (E) were immunoprecipitated with Cull-specific antibody, followed by western blotting with antibodies to Cul1 and ubiquitin. UB, ubiquitin.

Cell cycle analysis. Cells were fixed with $75 \%$ ethanol overnight at $-20^{\circ} \mathrm{C}$. After being washed twice with ice-cold PBS, the cells were incubated with RNase A $(100 \mu \mathrm{g} / \mathrm{ml})$ for $30 \mathrm{~min}$ at $37^{\circ} \mathrm{C}$ and then labeled with propidium iodide $(50 \mu \mathrm{g} / \mathrm{ml})$ for $15 \mathrm{~min}$. DNA contents were analyzed using a FACSCanto flow cytometer (BD Biosciences, Mississauga, ON, Canada).

Statistical analysis. All data were analyzed using the unpaired Student's t-test with GraphPad Prism 5 software. The data in this study are presented as means \pm standard deviation (SD). $\mathrm{P}<0.05$ was considered to indicate a statistically significant result.

\section{Results}

Cull promotes the ubiquitination and degradation of DEPTOR. To investigate the effect of Cull on DEPTOR expression, we stably overexpressed Cul1 in the A375 and Mewo cells and found that Cull overexpression suppressed the expression of DEPTOR (Fig. 1A). We next determined the effect of Cull depletion on DEPTOR levels and found that both Cul1-depleted A375 and Mewo cells had higher levels of DEPTOR than the controls (Fig. 1B). These results indicated an inversely correlated expression pattern between Cul1 and DEPTOR in melanoma cells. In addition, the effect of Cul1 on DEPTOR was suppressed in the presence of the proteasome inhibitor MG132 (Fig. 1C), thereby suggesting that the ubiquitin-proteasome pathway may be required for Cul1mediated reduction of DEPTOR protein abundance. Given that Cull serves as a rigid scaffold in the SCF complex and
DEPTOR is degraded via the ubiquitin-proteasome pathway by SCF ${ }^{\beta T r C P}$ E3 ubiquitin ligase, we detected the effect of Cull on the ubiquitination and degradation of DEPTOR. As shown in Fig. 1D, Cul1 overexpression promoted the ubiquitination of DEPTOR, whereas Cull depletion inhibited the ubiquitination of DEPTOR (Fig. 1E). Taken together, these results suggest that Cull decreases the expression of DEPTOR by promoting the ubiquitination and degradation of DEPTOR.

Cull enhances mTORCl activity by inhibiting the expression of DEPTOR. We demonstrated that Cull negatively regulates the expression of DEPTOR. Since DEPTOR inhibits mTORC1 activity, we speculated that Cul1 positively regulates mTORC1 activity. To test this hypothesis, the phosphorylation levels of 4E-BP1 and p70S6K, two downstream substrates of mTORC1, were detected in the control and Cull-overexpressing melanoma cell lines (A375 and Mewo). As shown in Fig. 2A, Cul1 overexpression promoted the phosphorylation of 4E-BP1 and p70S6K, indicating that Cull overexpression enhances mTORC1 activity. To confirm the positive effect of Cull on mTORC1 activity, we further determined the phosphorylation levels of 4E-BP1 and p70S6K in the control and Cul1-depleted melanoma cell lines (A375 and Mewo) and found that Cull knockdown profoundly attenuated the phosphorylation of 4E-BP1 and p70S6K (Fig. 2B). To investigate whether the negative effect of Cul1 depletion on mTORC1 activity results from DEPTOR accumulation, we silenced the expression of DEPTOR in the control and Cul1-depleted A375 cells and analyzed the phosphorylation of 4E-BP1 and p70S6K. As shown in Fig. 2C, the inhibitory effect of Cull depletion on 
A

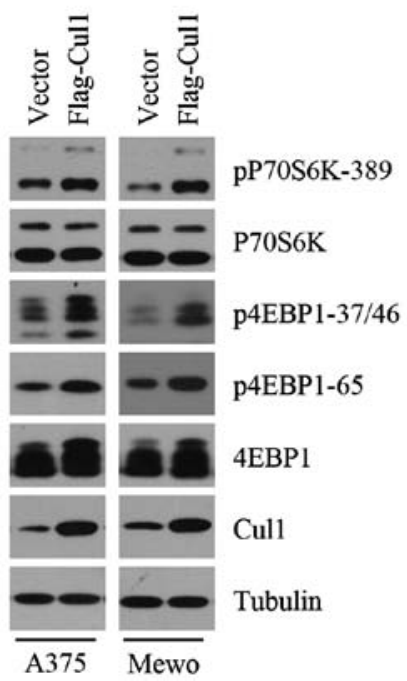

B

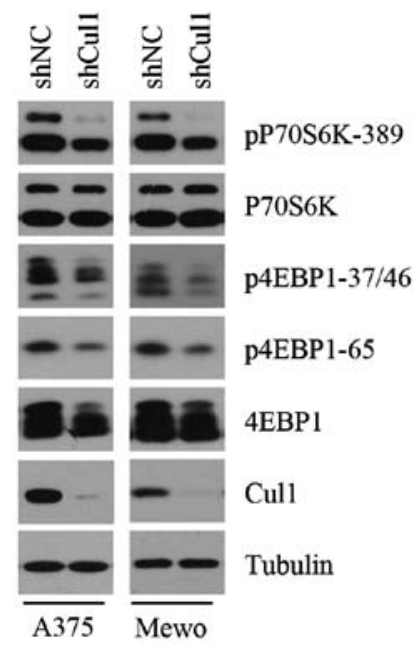

$\mathrm{C}$ si-DEPTOR $-\quad+\quad+$
shCull $-+\quad+$

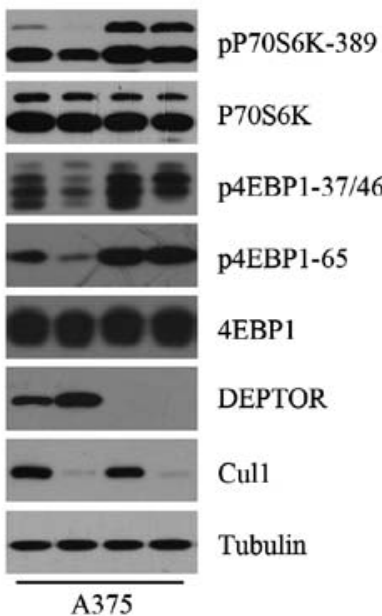

Figure 2. Cul1 enhances mTORC1 activity by inhibiting the expression of DEPTOR. (A) Cell lysates of control and melanoma cells (A375 and Mewo) stably transfected with FLAG-tagged Cull were analyzed by immunoblotting using the indicated antibodies. Tubulin was used as a loading control. (B) Cell lysates of control and Cul1-depleted melanoma cells (A375 and Mewo) were analyzed by immunoblotting using the indicated antibodies. Tubulin was used as a loading control. (C) Control and Cull-depleted A375 cells were transfected with control siRNA or DEPTOR siRNAs. After 48 h, the cells were collected and immunoblotted with the indicated antibodies. Tubulin was used as a loading control.

the phosphorylation of 4E-BP1 and p70S6K was rescued when DEPTOR was silenced, suggesting that Cull regulates mTORC1 activity in a DEPTOR-dependent manner. Taken together, these results suggest that Cull enhances mTORC1 activity by inhibiting the expression of DEPTOR.

Cull activates cap-dependent translation. It is well-known that mTORC1 promotes the formation of the eIF4F complex and activates cap-dependent translation by phosphorylating 4E-BP1 and relieving its binding to eIF4E (20). As we found that Cul1 enhanced the phosphorylation of 4E-BP1, we next investigated whether Cull enhances cap-dependent translation. To explore the function of Cul1 in cap-dependent translation, the effect of Cull on the assembly of the eIF4F complex was determined using 7-methyl GTP sepharose bead assay. The results show that Cull overexpression enhanced the interaction of eIF4E and eIF4G, while inhibiting the interaction of eIF4E and 4E-BP1 (Fig. 3A), indicating that Cul1 overexpression promotes the formation of the eIF4F complex. To confirm this result, we further detected the assembly of the eIF4F complex in the control and Cul1-depleted melanoma cells (A375 and Mewo) and found that Cull knockdown profoundly suppressed the interaction of eIF4E and eIF4G (Fig. 3B). Given that cap-dependent translation is dependent on the formation of the eIF4F complex, we next detected the effect of Cull on cap-dependent translation in the melanoma cells using a bicistronic luciferase reporter plasmid that detects cap-dependent translation of the Renilla luciferase gene and cap-independent Polio IRES-mediated translation of the firefly luciferase gene. The results showed that Cull overexpression activated cap-dependent translation (Fig. 3C and D), whereas knockdown of Cul1 inhibited cap-dependent translation in both the A375 and Mewo cells (Fig. 3E and F). To summarize, these findings suggest that Cull enhances the formation of the eIF4F complex, thus activating cap-dependent translation.
Cull promotes melanoma cell proliferation by activating cap-dependent translation. A previous study demonstrated that Cull enhances melanoma cell proliferation by promoting G1-S phase transition (10). However, the molecular mechanism behind this is not clearly understood. Cap-dependent translation plays a critical role in the control of cancer cell proliferation by initiating translation of cell cycle progressionrelated mRNAs, such as cyclin D1. Consistent with the positive effect of Cull on cap-dependent translation and previous results, we found that Cull overexpression enhanced the expression of cyclin D1, the percentage of cells in the $S$ phase and cell proliferation of melanoma cells. PP242, an mTOR kinase inhibitor, inhibited cap-dependent translation by decreasing the phosphorylation of 4E-BP1. 4EGI-1 suppressed cap-dependent translation initiation by disrupting the interaction of eIF4E and eIF4G. The results showed that either PP242 or 4EGI-1 treatment markedly reduced the promotive effect of Cull overexpression on cap-dependent translation in the A375 cells (Fig. 4A). To determine whether the positive effects of Cull overexpression on cell proliferation of melanoma cells are dependent on increased cap-dependent translation, control and Cull-overexpressing A375 cells were treated with PP242 or 4EGI-1 for the indicated times and then the expression of cyclin D1, the percentage of $\mathrm{S}$ phase cells and cell proliferation in the melanoma cells were detected. As shown in Fig. 4B-D, the promotive effects of Cull overexpression on cyclin D1 expression, the percentage of $\mathrm{S}$ phase cells and cell proliferation in the A375 cells were profoundly attenuated upon PP242 or 4EGI-1 treatment. Taken together, Cul1 promotes melanoma cell proliferation by activating cap-dependent translation.

4E-BP1 mediates the effects of Cull on cap-dependent translation and cell proliferation. $4 \mathrm{E}-\mathrm{BP} 1$ has been reported to negatively regulate cell proliferation by selectively inhibiting the translation of mRNA-encoding proteins involved 
A

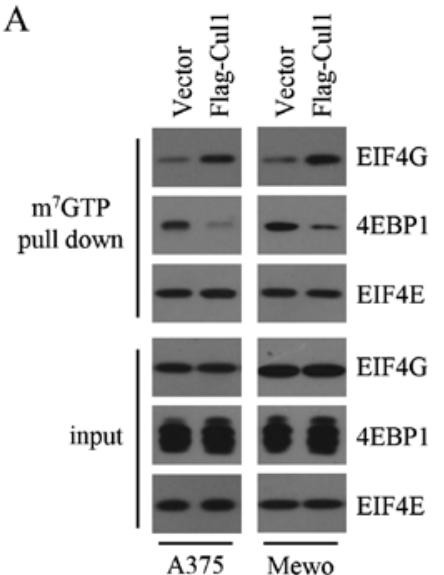

C

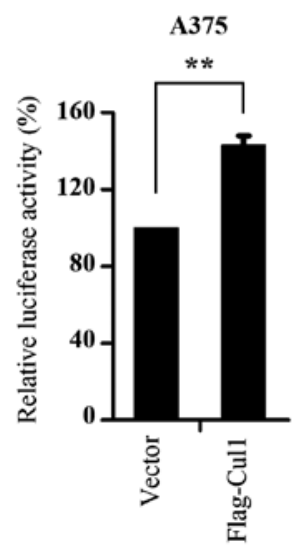

D

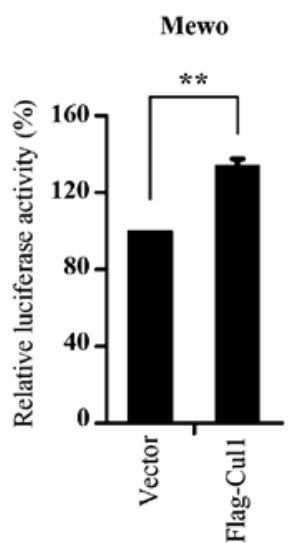

B

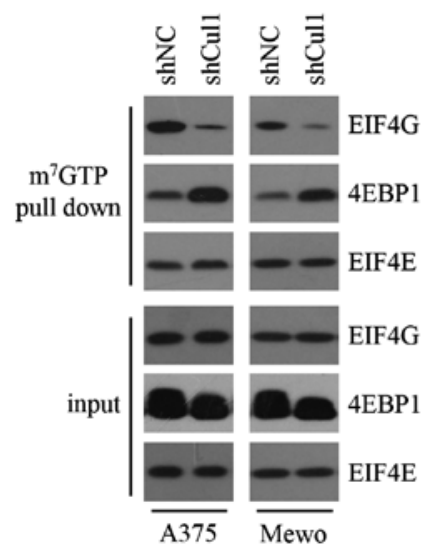

E

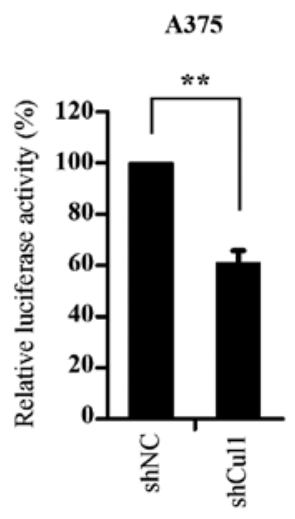

F

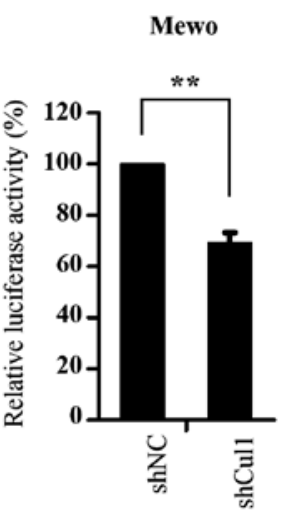

Figure 3. Cull activates cap-dependent translation. (A and B) $\mathrm{m}^{7}$ GTP pull down assay. Cell lysates from A375 and Mewo cells stably expressing FLAG-tagged Cul1 (A) or Cul1-depleted A375 and Mewo cells (B) were immunoblotted with the indicated antibodies or precipitated with $\mathrm{m}^{7} \mathrm{GTP}$ sepharose beads followed by western blotting with antibodies to eIF4G, 4E-BP1 and eIF4E. (C-F) Bicistronic luciferase assays. (C) A375 and (D) Mewo cells stably expressing FLAGtagged Cul1 or (E) Cul1-depleted A375 and (F) Mewo cells were transfected with a bicistronic luciferase reporter plasmid. Forty-eight hours post-transfection, luciferase activities were measured by a dual-luciferase assay. Cap-dependent translational activity was determined by calculating the ratio of Renilla/firefly luciferase luminescence. Data are presented as means $\pm \operatorname{SD}(n=3) .{ }^{* *} \mathrm{P}<0.01$.

in cell cycle progression, whereas $\mathrm{S} 6 \mathrm{~K}$ regulates cell size in mammalian cells (21). We demonstrated that Cul1 promotes melanoma cell proliferation by activating cap-dependent translation. To further confirm this result and determine the importance of 4E-BP1 dephosphorylation in mediating the effects of Cul1 depletion on cap-dependent translation and cell proliferation of melanoma cells, the expression of 4E-BP1 was silenced in the control and Cull-depleted A375 cells, and then cap-dependent translation, the expression of cyclin D1, the percentage of $\mathrm{S}$ phase cells and cell proliferation in the melanoma cells were detected. In agreement with the inhibitory effect of Cul1 depletion on 4E-BP1 phosphorylation, Cul1 knockdown decreased cap-dependent translation, the expression of cyclin D1, the percentage of S phase cells and cell proliferation in the A375 cells. However, 4E-BP1 depletion significantly reversed the inhibitory effect of the silencing of Cul1 on these processes (Fig. 5), suggesting that 4E-BP1 dephosphorylation is essential for Cull depletion to inhibit cap-dependent translation and cell proliferation. Taken together, these data suggest that 4E-BP1 mediates the effects of Cull on cap-dependent translation and cell proliferation in melanoma cells.

\section{Discussion}

Cull expression in melanoma tissues is profoundly upregulated compared with that in paired normal tissues, and increased Cull expression enhances melanoma cell proliferation by promoting G1-to-S phase transition, which is consistent with its first defined function as a regulator of the G1-to-S phase transition in budding yeast (22). As a scaffold protein, Cul1 binds to an adaptor protein SKP1 and an F-box protein at the $\mathrm{N}$-terminus and a RING protein $\mathrm{RBX} 1$ or $\mathrm{RBX} 2$ at the C-terminus to constitute the functional SCF E3 ligases. $\mathrm{SCF}^{\beta \mathrm{TrCP}}$, one of the SCF E3 ligases, has been reported to degrade DEPTOR (19). Given that aberrant expression of Cul1 is associated with dysfunction of SCF E3 ligases and decreased DEPTOR promotes cell proliferation via activating mTORC1, in this study we first determined the effect of Cull on DEPTOR expression levels. The results showed that the expression level of Cul1 was conversely associated with that of DEPTOR, suggesting the involvement of Cul1 in DEPTOR turnover. To verify this assumption, we investigated the effect of Cull on DEPTOR ubiquitination and degradation and found that Cul1 promoted the ubiquitination of DEPTOR, while 

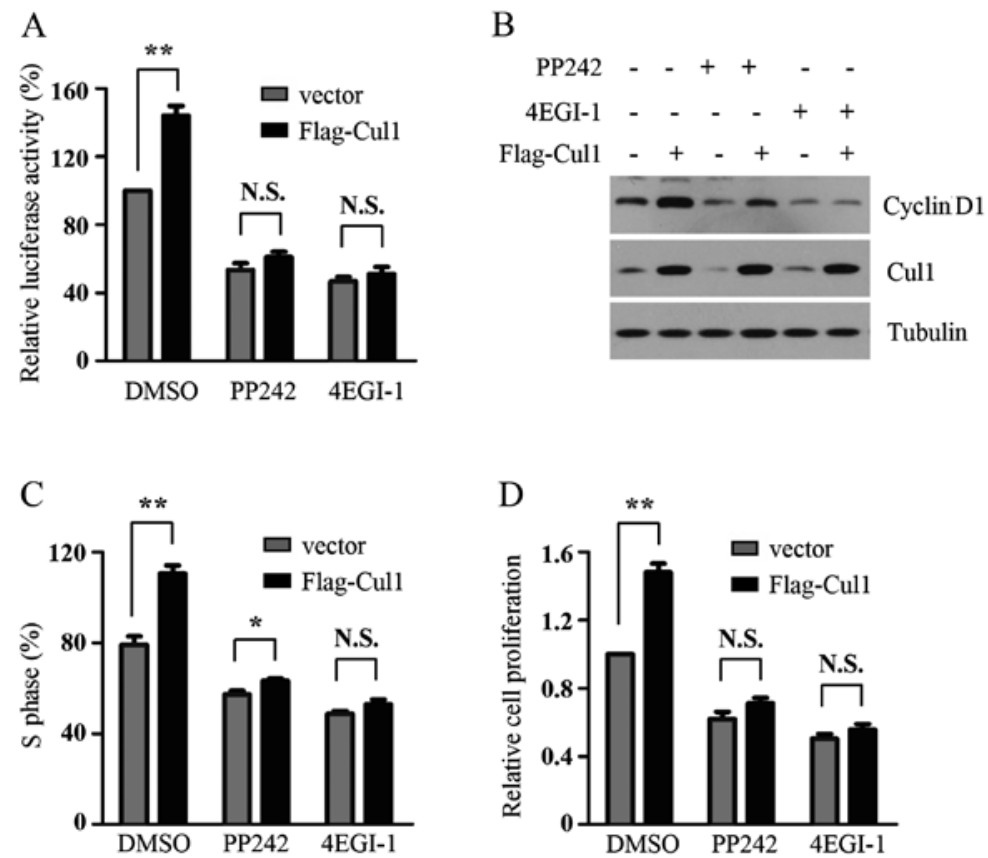

Figure 4. Cul1 promotes melanoma cell proliferation by activating cap-dependent translation. (A) Control and A375 cells stably transfected with FLAG-tagged Cul1 were transfected with a bicistronic luciferase reporter plasmid. Twenty-four hours post-transfection, the transfected cells were treated with $0.25 \mu \mathrm{M}$ PP242 or $50 \mu \mathrm{M} 4 \mathrm{EGI}-1$ for $24 \mathrm{~h}$ followed by bicistronic luciferase assays. Results are shown as means $\pm \mathrm{SD}\left(\mathrm{n}=3\right.$ ). ${ }^{* * *} \mathrm{P}<0.01$; N.S., not significant. (B) Control and A375 cells stably transfected with FLAG-tagged Cul1 were treated with $0.25 \mu \mathrm{M}$ PP242 or $50 \mu \mathrm{M} 4 \mathrm{EGI}-1$ for $24 \mathrm{~h}$ and then harvested for western blot analysis using the indicated antibodies. (C) Control and A375 cells stably transfected with FLAG-tagged Cul1 were treated as in B. The percentage of S phase cells was measured by flow cytometry. Data are presented as means $\pm \mathrm{SD}(\mathrm{n}=3)$. ${ }^{*} \mathrm{P}<0.05 ;{ }^{* *} \mathrm{P}<0.01$; N.S., not significant. (D) Control and A375 cells stably transfected with FLAG-tagged Cul1 were seeded in 96-well plates. After $24 \mathrm{~h}$, they were treated with DMSO, $0.25 \mu \mathrm{M}$ PP 242 or $50 \mu \mathrm{M} 4 \mathrm{EGI}-1$ for $48 \mathrm{~h}$. Cell proliferation was determined using the CCK-8 assay. Results are shown as means $\pm \mathrm{SD}(\mathrm{n}=3) .{ }^{* *} \mathrm{P}<0.01$; N.S., not significant.

A

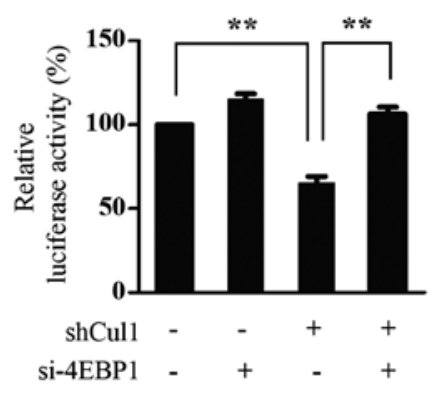

$\mathrm{C}$

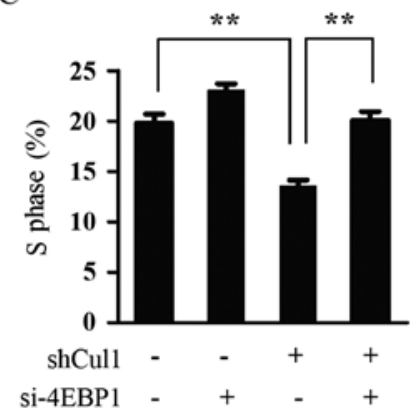

B

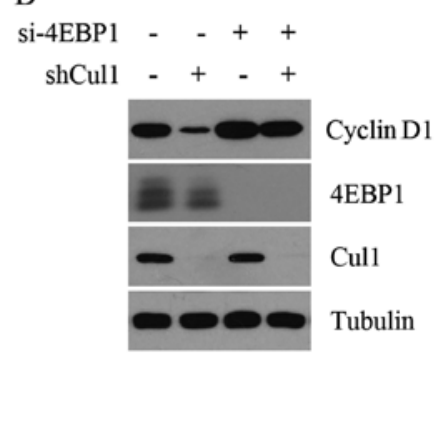

$\mathrm{D}$

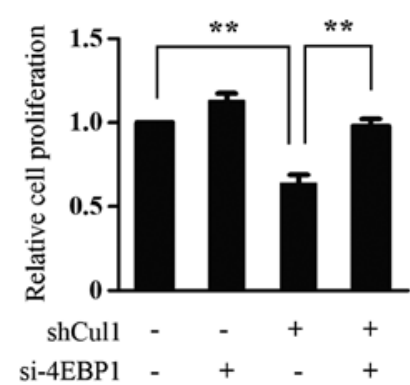

Figure 5. 4E-BP1 mediates the effects of Cul1 on cap-dependent translation and cell proliferation. (A) Control and Cul1-depleted A375 cells were transfected with a bicistronic luciferase reporter plasmid and control siRNA or 4E-BP1 siRNAs. Forty-eight hours post-transfection, luciferase activities were measured by a dual-luciferase assay. Results are shown as means $\pm \mathrm{SD}(\mathrm{n}=3) .{ }^{* *} \mathrm{P}<0.01$. (B) Control and Cul1-depleted A375 cells were transfected with control siRNA or 4E-BP1 siRNAs. After $48 \mathrm{~h}$, the cells were collected and immunoblotted with the indicated antibodies. Tubulin was used as a loading control. (C) Control and Cul1-depleted A375 cells were transfected with control siRNA or 4E-BP1 siRNAs. The percentage of S phase cells was measured by flow cytometry. Results are shown as means $\pm \mathrm{SD}(\mathrm{n}=3)$. ${ }^{* *} \mathrm{P}<0.01$. (D) Control and Cul1 depleted A375 cells were transfected with control siRNA or 4E-BP1 siRNAs. Twenty-four hours post-transfection, the cells were seeded in 96 -well plates. After $48 \mathrm{~h}$, cell proliferation was determined using the CCK-8 assay. Results are shown as means $\pm \mathrm{SD}(\mathrm{n}=3) .{ }^{* *} \mathrm{P}<0.01$. 
MG132, a proteasome inhibitor, blocked its promotive effect on the degradation of DEPTOR, suggesting that Cul1 decreases the expression of DEPTOR by promoting the ubiquitination and degradation of DEPTOR. Whether the expression level of Cul1 is conversely associated with that of DEPTOR in clinical tissues remains to be addressed and is a research direction we are currently pursuing.

DEPTOR expression negatively correlates with tumor progression in many cancers, including colorectal cancer and pancreatic ductal adenocarcinoma $(23,24)$. As a naturally occurring inhibitor of mTORC1, DEPTOR negatively regulates cell cycle progression and cell proliferation via suppressing mTORC1 activity. As we found that Cul1 inhibited the expression of DEPTOR, we next analyzed the effect of Cull on mTORC1 activity. We found that Cul1 enhanced mTORC1 activity by inhibiting the expression of DEPTOR. 4E-BP1 and p70S6K, two downstream substrates of mTORC1, have been reported to regulate cell proliferation and cell size in mammalian cells, respectively (21). As we aimed to investigate the underlying mechanisms involved in the regulation of melanoma cell proliferation by Cul1, our subsequent research focused on 4E-BP1. 4E-BP1 negatively regulates the formation of the eIF4F complex and cap-dependent translation by competing with eIF4G for binding to eIF4E. Upon being phosphorylated by mTORC1, 4E-BP1 relieves its binding to eIF4E, permitting the assembly of the eIF4F complex to initiate cap-dependent translation. Activation of mTORC1 was reported to be strongly associated with malignant melanocytic lesions in vivo and inhibition of mTORC1 activity using rapamycin suppressed the proliferation of melanoma-derived cell lines $(25,26)$. Hyperphosphorylated 4E-BP1 was reported to be associated with worse overall and post-recurrence survival of metastatic melanoma patients (13). The expression of eIF4E is strongly elevated in melanoma and positively correlated with that of VEGF and cyclin D1 (27), the mRNAs of which are translated in a cap-dependent translation manner. These studies indicate that cap-dependent translation plays a key role in melanoma development. Since we found that Cul1 activated mTORC1 and enhanced the phosphorylation of 4E-BP1 in melanoma cells, we speculated that Cull may activate cap-dependent translation. Using 7-methyl GTP sepharose bead and bicistronic luciferase reporter assays, we validated this hypothesis.

Cap-dependent translation promotes cell proliferation through initiating the translation of mRNA encoding proteins involved in cell cycle progression, such as cyclin D1 and c-myc. Therefore, it is reasonable to speculate that Cul1 promotes melanoma cell proliferation by activating cap-dependent translation. Our results showed that blocking cap-dependent translation using PP242 or 4EGI-1 profoundly attenuated the promotive effects of Cull overexpression on the expression of cyclin D1, the percentage of $\mathrm{S}$ phase cells and cell proliferation in melanoma cells. Subsequently, we aimed to ascertain the role of 4E-BP1 phosphorylation in mediating the effects of Cull on cap-dependent translation and cell proliferation of melanoma cells. Cul1 depletion dephosphorylates 4E-BP1 and promotes $4 \mathrm{E}-\mathrm{BP} 1$ to compete with eIF4G for binding to eIF4E, leading to the inhibition of cap-dependent translation. We relieved the sequestered eIF4E by hypophosphorylated $4 \mathrm{E}-\mathrm{BP} 1$ by silencing the expression of 4E-BP1 in control and Cul1-depleted A375 cells and found that silencing of 4E-BP1 significantly reversed the suppressive effect of the silencing of Cull on cap-dependent translation and cell proliferation. However, 4E-BP1 knockdown did not completely restore capdependent translation and cell proliferation suppressed by Cull depletion, suggesting that Cull may regulate other molecules involved in cell proliferation in melanoma cells. In support of this, Cul1 has been reported to regulate cell proliferation by decreasing the expression of p27 in many types of cancer cells, including melanoma cells $(6,7,10)$.

In summary, we found that Cul1 promoted cap-dependent translation and melanoma cell proliferation by promoting DEPTOR degradation and activating mTORC1. Cul1 acts as a scaffold to constitute the intact SCF E3 ligases, consisting of 4 functional components: a substrate-recognizing F-box protein, an adaptor protein SKP1, a scaffold protein Cul1, a RING protein RBX1 or RBX2 (28). In addition to Cul1, other components of SCF E3 ligases have been reported to be implicated in skin cancers. RBX2, a RING protein, was reported to promote the development of skin cancer by promoting inhibitor of $\mathrm{I} \kappa \mathrm{B} \alpha$ degradation to activate NF- $\kappa \mathrm{B}(29)$. The expression of SKP2, an F-box protein, is gradually increased during melanomagenesis from melanocytic nevi to metastatic melanoma and is associated with a poorer 5-year survival of melanoma patients (30). SKP2 promotes cell proliferation by targeting the degradation of p27 and ING3 $(31,32) . \beta \operatorname{TrCP} 1$ and $\beta \operatorname{TrCP} 2$, two F-box proteins, are overexpressed in DMBA/TPA-induced mouse skin papillomas and contribute to skin papillomagenesis by accelerating degradation of $\mathrm{I} \kappa \mathrm{B} \alpha(29,33)$. These studies demonstrate that SCF E3 ubiquitin ligases play a critical role in skin carcinogenesis and represent a potential therapeutic target for the treatment of human skin cancer. In fact, MLN4924, an indirect inhibitor of SCF E3 ligases, was shown to be well tolerated and effective in patients with metastatic melanoma in phase I clinical trials (34). In addition, our results indicate that Cull promotes melanoma cell proliferation by activating cap-dependent translation, demonstrating that targeting mTORC1 or the eIF4F complex may be effective for the treatment of melanoma patients with elevated Cull expression.

\section{Acknowledgements}

The present study was supported by the Guizhou Province Chinese Native Medicine Modernization Special Project (20125018 to Y.C.) and Guiyang Science and Technology Bureau Science and Technology Innovation Platform Project (2012303 to Y.C.).

\section{References}

1. Sosman JA, Kim KB, Schuchter L, Gonzalez R, Pavlick AC, Weber JS, McArthur GA, Hutson TE, Moschos SJ, Flaherty KT, et al: Survival in BRAF V600-mutant advanced melanoma treated with vemurafenib. N Engl J Med 366: 707-714, 2012.

2. Liu W, Wang Y, Zhang C, Huang B, Bai J and Tian L: Cullin1 is up-regulated and associated with poor patients' survival in hepatocellular carcinoma. Int J Clin Exp Pathol 8: 4001-4007, 2015.

3. Wang W, Chen Y, Deng J, Zhou J, Gu X, Tang Y, Zhang G, Tan Y, Ge Z, Huang Y, et al: Cullin1 is a novel prognostic marker and regulates the cell proliferation and metastasis in colorectal cancer. J Cancer Res Clin Oncol 141: 1603-1612, 2015.

4. Fan YC, Zhu YS, Mei PJ, Sun SG, Zhang H, Chen HF, Chen C and Miao FA: Cullin1 regulates proliferation, migration and invasion of glioma cells. Med Oncol 31: 227, 2014. 
5. Xu M, Yang X, Zhao J, Zhang J, Zhang S, Huang H, Liu Y and Liu J: High expression of Cullin 1 indicates poor prognosis for NSCLC patients. Pathol Res Pract 210: 397-401, 2014.

6. Bai J, Yong HM, Chen FF, Mei PJ, Liu H, Li C, Pan ZQ, Wu YP and Zheng JN: Cullin 1 is a novel marker of poor prognosis and a potential therapeutic target in human breast cancer. Ann Oncol 24: 2016-2022, 2013.

7. Bai J, Zhou Y, Chen G, Zeng J, Ding J, Tan Y, Zhou J and Li G: Overexpression of Cullin1 is associated with poor prognosis of patients with gastric cancer. Hum Pathol 42: 375-383, 2011.

8. Chen G, Cheng Y, Martinka M and Li G: Cul1 expression is increased in early stages of human melanoma. Pigment Cell Melanoma Res 23: 572-574, 2010.

9. Zhang G and Li G: Novel multiple markers to distinguish melanoma from dysplastic nevi. PLoS One 7: e45037, 2012.

10. Chen $\mathrm{G}$ and Li G: Increased Cull expression promotes melanoma cell proliferation through regulating p27 expression. Int $\mathrm{J}$ Oncol 37: 1339-1344, 2010.

11. Spilka R, Ernst C, Mehta AK and Haybaeck J: Eukaryotic translation initiation factors in cancer development and progression. Cancer Lett 340: 9-21, 2013.

12. Kong $\mathbf{J}$ and Lasko P: Translational control in cellular and developmental processes. Nat Rev Genet 13: 383-394, 2012.

13. O'Reilly KE, Warycha M, Davies MA, Rodrik V, Zhou XK, Yee H, Polsky D, Pavlick AC, Rosen N, Bhardwaj N, et al: Phosphorylated 4E-BP1 is associated with poor survival in melanoma. Clin Cancer Res 15: 2872-2878, 2009.

14. Chiarini F, Evangelisti C, McCubrey JA and Martelli AM: Current treatment strategies for inhibiting mTOR in cancer. Trends Pharmacol Sci 36: 124-135, 2015.

15. Zoncu R, Efeyan A and Sabatini DM: mTOR: From growth signal integration to cancer, diabetes and ageing. Nat Rev Mol Cell Biol 12: 21-35, 2011.

16. Wang Z, Zhong J, Inuzuka H, Gao D, Shaik S, Sarkar FH and Wei W: An evolving role for DEPTOR in tumor development and progression. Neoplasia 14: 368-375, 2012.

17. Duan S, Skaar JR, Kuchay S, Toschi A, Kanarek N, Ben-Neriah Y and Pagano M: mTOR generates an auto-amplification loop by triggering the $\beta \operatorname{TrCP}-$ and $\mathrm{CK} 1 \alpha$-dependent degradation of DEPTOR. Mol Cell 44: 317-324, 2011.

18. Gao D, Inuzuka H, Tan MK, Fukushima H, Locasale JW, Liu P, Wan L, Zhai B, Chin YR, Shaik S, et al: mTOR drives its own activation via $\mathrm{SCF}(\beta \operatorname{TrCP})$-dependent degradation of the mTOR inhibitor DEPTOR. Mol Cell 44: 290-303, 2011

19. Zhao Y, Xiong X and Sun Y: DEPTOR, an mTOR inhibitor, is a physiological substrate of $\mathrm{SCF}(\beta \operatorname{TrCP}) \mathrm{E} 3$ ubiquitin ligase and regulates survival and autophagy. Mol Cell 44: 304-316, 2011.

20. Silvera D, Formenti SC and Schneider RJ: Translational contro in cancer. Nat Rev Cancer 10: 254-266, 2010.
21. Dowling RJ, Topisirovic I, Alain T, Bidinosti M, Fonseca BD, Petroulakis E, Wang X, Larsson O, Selvaraj A, Liu Y, et al: mTORC1-mediated cell proliferation, but not cell growth, controlled by the 4E-BPs. Science 328: 1172-1176, 2010.

22. Kipreos ET, Lander LE, Wing JP, He WW and Hedgecock EM: cul-1 is required for cell cycle exit in C.elegans and identifies a novel gene family. Cell 85: 829-839, 1996.

23. Lai EY, Chen ZG, Zhou X, Fan XR, Wang H, Lai PL, Su YC, Zhang BY, Bai XC and Li YF: DEPTOR expression negatively correlates with mTORC1 activity and tumor progression in colorectal cancer. Asian Pac J Cancer Prev 15: 4589-4594, 2014.

24. Li H, Sun GY, Zhao Y, Thomas D, Greenson JK, Zalupski MM, Ben-Josef E and Sun Y: DEPTOR has growth suppression activity against pancreatic cancer cells. Oncotarget 5: 12811-12819, 2014.

25. Pópulo H, Soares $\mathrm{P}$ and Lopes JM: Insights into melanoma: Targeting the mTOR pathway for therapeutics. Expert Opin Ther Targets 16: 689-705, 2012.

26. Marone R, Erhart D, Mertz AC, Bohnacker T, Schnell C, Cmiljanovic V, Stauffer F, Garcia-Echeverria C, Giese B, Maira SM, et al: Targeting melanoma with dual phosphoinositide 3-kinase/mammalian target of rapamycin inhibitors. Mol Cancer Res 7: 601-613, 2009.

27. Yang SX, Hewitt SM, Steinberg SM, Liewehr DJ and Swain SM: Expression levels of eIF4E, VEGF, and cyclin D1, and correlation of eIF4E with VEGF and cyclin D1 in multi-tumor tissue microarray. Oncol Rep 17: 281-287, 2007.

28. Jia L and Sun Y: SCF E3 ubiquitin ligases as anticancer targets. Curr Cancer Drug Targets 11: 347-356, 2011.

29. Gu Q, Bowden GT, Normolle D and Sun Y: SAG/ROC2 E3 ligase regulates skin carcinogenesis by stage-dependent targeting of c-Jun/AP1 and IkappaB-alpha/NF-kappaB. J Cell Biol 178: 1009-1023, 2007.

30. Chen G, Cheng Y, Zhang Z, Martinka M and Li G: Cytoplasmic Skp2 expression is increased in human melanoma and correlated with patient survival. PLoS One 6: e17578, 2011.

31. Li Q, Murphy M, Ross J, Sheehan C and Carlson JA: Skp2 and p27 ${ }^{\text {kipl }}$ expression in melanocytic nevi and melanoma: An inverse relationship. J Cutan Pathol 31: 633-642, 2004

32. Chen G, Wang Y, Garate M, Zhou J and Li G: The tumor suppressor ING3 is degraded by SCF(Skp2)-mediated ubiquitin-proteasome system. Oncogene 29: 1498-1508, 2010.

33. Bhatia N, Herter JR, Slaga TJ, Fuchs SY and Spiegelman VS: Mouse homologue of HOS (mHOS) is overexpressed in skin tumors and implicated in constitutive activation of NF-kappaB. Oncogene 21: 1501-1509, 2002

34. Nawrocki ST, Griffin P, Kelly KR and Carew JS: MLN4924: A novel first-in-class inhibitor of NEDD8-activating enzyme for cancer therapy. Expert Opin Investig Drugs 21: 1563-1573, 2012. 